\title{
La CAN et le MERCOSUR : bilan et perspectives
}

La CAN y el MERCOSUR: balance y perspectivas

The CAN and the MERCOSUR: appraisal and perspective

\section{Axel Gastambide}

\section{(2) OpenEdition}

\section{Journals}

Édition électronique

URL : https://journals.openedition.org/bifea/7071

DOI : 10.4000/bifea.7071

ISSN : 2076-5827

Éditeur

Institut Français d'Études Andines

Édition imprimée

Date de publication : 1 août 2001

Pagination : 233-263

ISSN : 0303-7495

Référence électronique

Axel Gastambide, « La CAN et le MERCOSUR : bilan et perspectives », Bulletin de I'Institut français d'études andines [En ligne], 30 (2) | 2001, mis en ligne le 08 août 2001, consulté le 21 septembre 2021. URL : http://journals.openedition.org/bifea/7071 ; DOI : https://doi.org/10.4000/bifea.7071

Les contenus du Bulletin de l'Institut français d'études andines sont mis à disposition selon les termes de la licence Creative Commons Attribution - Pas d'Utilisation Commerciale - Pas de Modification 4.0 International. 


\title{
LA CAN ET LE MERCOSUR : BILAN ET PERSPECTIVES
}

\author{
Axel GASTAMBIDE*
}

\section{Résumé}

L'Amérique du sud n'a pas échappé au processus de régionalisation commerciale datant du début des années 1990. Le commerce entre les pays andins, dans le cadre de la CAN, et celui des pays du cône sud, dans le cadre du MERCOSUR, a augmenté de manière spectaculaire depuis dix ans. Parallèlement, le nord du continent s'est regroupé, avec succès, autour d'un accord commercial préférentiel, l'ALENA.

Actuellement, à l'initiative des États-Unis, un projet vise à la création d'une zone de libre échange continentale en 2005. Si les pays sud-américains sont ouverts à l'idée d'un meilleur accès aux marchés régionaux, ils craignent que les États-Unis n'imposent un accord "OMC plus" (contenant des clauses sur le droit au travail et sur l'environnement) qui pénaliserait leur économie. Ainsi, afin de constituer un bloc unitaire et cohérent, susceptible de peser dans les négociations, la CAN et le MERCOSUR ont prévu, pour 2002, l'instauration d'une zone de libre échange en Amérique du sud.

Mots clés : Accord commercial, CAN, MERCOSUR, ZLEA.

\section{LA CAN Y EL MERCOSUR: BALANCE Y PERSPECTIVAS}

\section{Resumen}

América del Sur no escapó del proceso de regionalismo comercial que data de inicios de la década de los 90 . El comercio entre los países andinos, en el marco de la CAN, y entre los países pertenecientes al cono sur, en el marco del MERCOSUR, ha aumentado de manera espectacular en los últimos diez años. Paralelamente, el norte del continente se ha reagrupado alrededor de un acuerdo comercial preferencial, el llamado ALENA, cuyo éxito no se ha desdicho.

Actualmente, bajo la inicitativa de los Estados Unidos, se avisora un proyecto para el 2005: la creación de una zona de librecambio para el continente. Por un lado, los países sudamericanos ocuparán una posición favorable en lo que se refiere a un mejor acceso a los mercados regionales, pero, por otro lado, ellos temen que los EEUU impondrán un acuerdo "OMC plus" (el mismo que debería contener las cláusulas sobre el derecho al trabajo y sobre el medio ambiente) que perjudicaría su economía. De esta manera, a fin de constituir un bloque

* Centre d'Études et de Recherches sur le Développement International (CERDI), Université d'Auvergne. E-mail : A.Gastambide@u-clermont1.fr

L'auteur remercie Olivier Dabène, Sylviane Guillaumont, Samuel Guérineau et Yves SaintGeours pour leurs remarques sur une version préliminaire. 
unitario y coherente, suceptible de hacer frente a las negociaciones, la CAN y el MERCOSUR han previsto, para el año 2002, la instauración de una zona librecambio en América del Sur.

Palabras claves: Acuerdo comercial, CAN, MERCOSUR, ZLEA.

\title{
THE CAN AND THE MERCOSUR: APPRAISAL AND PERSPECTIVE
}

\begin{abstract}
South America did not avoid the process of commercial regionalism in the beginning of the 1990's. Trade between Andean countries, within the framework of the CAN, and the one between Southern Cone countries, within the framework of the MERCOSUR, have dramatically been increasing over the last ten years. Meanwhile, the northern part of the continent regrouped with success around,, a preferential commercial agreement called ALENA.

At the present time, the United States promotes a project aiming at the creation in 2005 of a continental free trade area . South America's countries are, on one hand, favoured by better access to regional markets but, on the other hand, they fear that United States will enforce an agreement "OMC plus" (including clauses about labour right and environment) which would penalise their economy. So, in order to constitute a unitary and coherent block, likely to influence on negotiations, CAN and MERCOSUR have planned, for 2002, the creation of a free trade area in South America.
\end{abstract}

Key words: Commercial agreement, CAN, MERCOSUR, ZLEA.

Les années 1980 marquent un profond changement de doctrine économique en Amérique du sud: de l'étatisme industrialiste au libéralisme. Pour se protéger des conséquences de la crise de 1929, les économies de la région avaient progressivement adopté des stratégies de substitution aux importations axées sur la protection et la promotion du marché interne, politiques qui s'inscriront, plus tard, dans le consensus politique national. Cette stratégie de développement, en contribuant au développement de l'industrie nationale et en permettant une diversification économique, a surtout profité aux pays disposant d'un marché relativement grand (Argentine, Brésil, Colombie). Cependant, la forte protection a également entraîné, pour tous les pays, de profondes distorsions dans l'allocation des ressources et a limité l'efficacité du secteur industriel. Au début des années 1980, la crise de la dette a conduit les différents gouvernements à mettre en œuvre des politiques de réduction de la demande afin de dégager un excédent de la balance commerciale permettant de financer la partie des intérêts de la dette non couverte par l'endettement nouveau. Ces politiques de stabilisation, en bridant la croissance économique, ont accentué le marasme économique ; les déficits budgétaires se sont donc aggravés et ont provoqué une période de forte inflation et des épisodes d'hyper-inflation. Ces instabilités macro-économiques, combinées à l'application accrue, dans le monde, de politique économique d'inspiration libérale, ont entériné le rejet des politiques de substitution aux importations au profit de politiques structurelles libérales (1) reposant sur deux axes principaux, la libéralisation des prix et l'ouverture

(1) Le Chili et l'Uruguay se sont engagés dans une politique libérale dès 1973. 
sur l'extérieur (afin de permettre, selon les auteurs, une allocation plus efficiente des ressources). L'ouverture commerciale, en raison de son caractère drastique et généralisé, constitue la réforme la plus spectaculaire (Miotti et al.,1996).

Pour les pays sud-américains, la libéralisation commerciale vis à vis de l'extérieur s'organise de trois manières. Dans la logique du changement de politique économique, les pays de la région, à partir de 1987, ont d'abord libéralisé, unilatéralement les importations. L'objectif est de confronter les producteurs de biens importables à la concurrence internationale et d'encourager le secteur de biens exportables. Cette libéralisation unilatérale s'est accompagnée d'une autre, multilatérale, dans le cadre de l'Accord général sur les tarifs douaniers et le commerce (GATT) puis de l'Organisation Mondiale du Commerce (OMC) (2). La troisième forme s'est effectuée dans un cadre régional (3) à partir d'accords commerciaux entre économies sud-américaines (voir annexe 1).

Au début des années 1990, le Brésil, l’Argentine, le Paraguay et le Chili ont signé un accord commercial créant le Marché Commun du Sud (MERCOSUR) et la Bolivie, la Colombie, l'Équateur, le Pérou et le Venezuela ont renégocié en profondeur l'accord du Pacte andin (rebaptisé Communauté Andine des Nations, CAN, en 1996) datant de 1969. Depuis, le succès de ces deux unions commerciales ne s'est pas démenti. Sur la base de ces deux accords, depuis trois ans, des discussions se sont engagées en vue de constituer en 2002 une zone de libre échange (ZLE) dans la région.

Après avoir fait un bilan des deux accords mentionnés, nous analyserons le projet de libre échange sud-américain (CAN-MERCOSUR) dont la logique est déterminée, pour partie, par le vaste projetZone de Libre Échange des Amériques (ZLEA) entre tous les pays du continent.

\section{UN BILAN DES ACCORDS DE LA CAN ET DU MERCOSUR}

À l'instar de l'Europe, dès la fin de la seconde guerre mondiale, les pays d'Amérique du sud ont cherché à développer une coopération commerciale afin, notamment, de favoriser les politiques de substitution aux importations. En 1960, l'Association Latino-Américaine de Libre Échange (4) (ALALE) prévoyait la création d'une ZLE. L'accord reposait sur un multilatéralisme qui ne faisait pas avancer les échanges. L'échec de l'ALALE a favorisé, en 1969, un groupement sous-régional entre les pays andins (Pacte andin). En 1980, l'Association Latino-Américaine D'Intégration (ALADI) succède à l'ALALE et cherche à promouvoir le libre échange à travers des

(2) En 1996, après l'adhésion de l'Équateur à l'OMC, tous les pays d'Amérique du sud appartiennent à l'OMC.

(3) Il existe actuellement un vaste débat concernant la complémentarité (Éthier, 1998) ou substituabilité (Panagariya, 1996) du régionalisme et de l'uni/multilatéralisme. Selon Delvin \& Ffrench-Davis (1999), en Amérique du sud, la libéralisation uni/multilatérale a diminué les tarifs à un niveau relativement faible, si bien que l'effet du régionalisme ne remettrait pas en cause la politique uni/multilatérale d'ouverture (effet substitualité non significatif).

(4) L'ALALE comprenait l'Argentine, la Bolivie, le Brésil, le Chili, la Colombie, l'Équateur, le Guatemala, le Mexique, le Pérou et le Venezuela. 
relations intra-zone, même limitées à deux pays. C'est dans ce cadre que l'Argentine et le Brésil ont signé, en 1986, 12 protocoles commerciaux à l'origine du MERCOSUR.

\section{1. La Communauté Andine des Nations (ex-Pacte andin)}

\section{1. 1. Les premières années}

L'accord du Pacte andin est signé en 1969 à Cartagena par la Bolivie, la Colombie, le Chili, l'Équateur et le Pérou. L'objectif des signataires est de créer un espace commercial intégré afin de faciliter la mise en place d'une politique d'industrialisation équilibrée de la région (5).

L'accord comprend deux volets principaux. Le premier vise à la constitution d'une ZLE (suppression des barrières tarifaires et non tarifaires entre ses membres) et d'une union douanière (tarif extérieur commun vis à vis de l'extérieur [TEC]) au plus tard en 1980 pour le Chili, la Colombie et le Pérou, et en 1985 pour la Bolivie et l'Équateur, (bénéficiant, jusqu'à cette date, d'un régime préférentiel). Le deuxième axe concerne la politique industrielle. Elle est basée sur une politique de substitution aux importations traditionnelle (remplacement d'importation par une production intérieure) à laquelle s'ajoute la dimension régionale. Cette dernière, en augmentant la taille du marché local et en créant des externalités et des économies d'échelle, doit favoriser le processus d'industrialisation. Un organe planificateur, le Programme Sectoriel de Développement Industriel (PSDI) est chargé d'organiser la politique industrielle et de la rendre équitable afin de limiter les tensions au sein de l'accord.

Rapidement, les droits de douane dans la région diminuent fortement. Un TEC est adopté mais son application se heurte aux oppositions nationales, notamment chilienne. Un régime commun pour les capitaux étrangers est mis en place en 1971. L'année suivante, l'industrie métallurgique bénéficie du premier programme sectoriel. Mais, en 1973, l'adhésion du Venezuela oblige à une difficile révision de la politique industrielle. En 1976, aucun programme industriel n'est effectivement appliqué.

Entre 1971 et 1976, le commerce intra-zone passe de 111 millions de dollars en 1971 à 613 millions en 1976. Cette hausse du commerce est la plus forte pour les produits manufacturés non traditionnels, ce qui correspond aux objectifs initiaux de l'accord (Salgado, 1998). L'accord apparaît donc comme une réussite ; même s'il était attendu que le commerce augmente dans les premières années compte tenu de la forte protection en vigueur avant 1969. Mais, très vite les difficultés s'accumulent : le TEC n'est pas appliqué et la politique industrielle est un échec à cause, notamment, d'un manque d'efficacité des mécanismes de compensation.

\section{1. 2. 1976-1987: la crise}

En 1976, le Chili, engagé depuis 3 ans dans une politique libérale, quitte le Pacte andin. Cette rupture marque un coup d'arrêt dans le processus d'intégration. Les

(5) Dans cet accord, le commerce est clairement un instrument au service de l'objectif de développement économique par l'industrialisation. Il se différencie donc des accords commerciaux récents où la recherche d'une augmentation du commerce est une fin en soi. 
membres appliquent de moins en moins les décisions juridiques destinées à régler les conflits entre les partenaires. En 1979, les pays membres, conscients des difficultés, repoussent les dates butoirs de l'entrée en vigueur de la ZLE et du TEC : respectivement 1983 et 1984 pour la Colombie, le Pérou et le Venezuela et 1989 pour la Bolivie et l'Équateur (protocole d'Arequipa). Cependant, des instabilités politiques (conflit frontalier entre l'Équateur et le Pérou en 1981, coup d'État, la même année, du dictateur García Meza en Bolivie qui est, du même coup, exclue temporairement des organes communautaires du Pacte andin) rendent les avancées dans le processus d'intégration presque impossibles.

La crise de la dette des années 1980 porte un coup presque fatal à l'accord de Cartagena. Les économies de la région sont en récession et ne sont donc pas favorables à l'ouverture commerciale. En 1983, le programme de libéralisation est suspendu et un système dit de commerce administré le remplace. Des accords bilatéraux sont signés et de nombreux produits "sensibles" sont protégés. L’intégration commerciale des pays andins est dans les faits suspendue.

\section{1. 3. 1987-2000 : réactivation du Pacte andin}

En 1987, les pays membres tentent de sauver l'accord. Le protocole de Quito, dont les dispositions seront effectives à partir de 1989, modifie pour la première fois, en profondeur l'accord initial. Le Pacte andin abandonne sa dimension "industrialiste" et "developpementiste" pour se concentrer sur la promotion du commerce intra-zone. L'objectif prioritaire est la constitution d'une ZLE et l'adoption d'un TEC. Par ailleurs, la possibilité d'accords bilatéraux est maintenue (6).

Le début de la décennie 1990 marque le retour de la croissance dans la région et le processus d'intégration andin connaît des avancés spectaculaires. Depuis le 31 janvier 1993, la Bolivie, la Colombie, l'Équateur et le Venezuela forment une ZLE sans exception. Le Pérou suit un régime à part. En effet, ce pays a suspendu en 1992 son programme de libéralisation et l'a ensuite repris en 1997. Il doit intégrer la ZLE au plus tard en 2005.

Le 26 novembre 1994, la Bolivie, la Colombie, l'Équateur et le Venezuela adoptent un TEC qui entre en vigueur le $1^{\text {er }}$ février 1995. Il existe quatre tarifs selon les produits de 5,10, 15 et 20\%. Cependant, l'union douanière est partielle : l'Équateur et la Bolivie disposant d'un régime provisoire différencié (7). De plus, il existe deux listes d'exception: une première liste comprenant les biens et services liés à la santé, l'éducation et la culture et une seconde englobant des produits marchands (8) qui, en juin

(6) Cette disposition qui, a priori, est en contradiction avec l'objectif d'intégration régionale non discriminatoire est une concession à des économies andines réticentes à la libéralisation de leur commerce dans un contexte de crise économique.

(7) L’Équateur bénéficie d'un régime spécial autorisant une différence du tarif de 5 points par rapport à la référence de base. La Bolivie applique, vis à vis de l'extérieur, une politique tarifaire de 5 ou $10 \%$ qu'elle avait instaurée préalablement dans des dispositions commerciales unilatérales.

(8) En Colombie, en Équateur et au Venezuela, le tarif appliqué dans les secteurs de l'automobile et de la construction navale s'élève à $35 \%$. De plus, en Équateur, les produits miniers sont taxés à $99 \%$. 
2000, ne devait plus être soumise à ce régime spécial. Le marché commun, phase supérieure de l'intégration commerciale, devant assurer la libre circulation des biens, des services, des capitaux et des hommes, est prévu pour 2005 (9).

\section{2. Le Marché Commun du Sud (MERCOSUR)}

L'origine du MERCOSUR remonte à l'année 1986 lorsque l'Argentine et le Brésil signent un protocole d'intégration. D'autres accords bilatéraux sont ensuite signés entre ces deux pays, puis avec le Paraguay et l'Uruguay. Au début des années 1990, ces accords sont renégociés et aboutissent, le 26 mars 1991, à la signature du traité d'Asunción (par les quatre pays) marquant l'acte de naissance du bloc commercial du MERCOSUR.

La constitution du MERCOSUR peut trouver son explication par la conjonction de deux facteurs : la crise économique et le retour de la démocratie. La crise de la dette des années 1980 a suscité l'idée qu'un front uni des débiteurs pourrait créer un rapport de force nouveau concernant les négociations de la dette.

“Bien que cette perspective constituât plus une éventualité qu'un instrument réel, elle fut toujours présente en toile de fond des positions officielles de cette période." (Schvarzer, 1999 : 81)

Parallèlement, les quatre pays ont connu une transition démocratique après une longue période de dictature militaire (10). Les nouveaux dirigeants politiques ont cherché à créer des liens, notamment commerciaux, afin de prévenir le retour éventuel de coup d'État militaire. Dans ce but, les conventions d'intégration visèrent à éliminer les noyaux de conflits (notamment à travers une coopération nucléaire entre l'Argentine et le Brésil) qui avaient nourri par le passé une course aux armements et le repli sur soi. Selon la même logique, le traité d'Asunción stipule que la démocratie est une condition fondamentale pour appartenir au MERCOSUR et fait à tous ses signataires l'obligation de la défendre (11).

Entre 1986 et 1989, le processus d'intégration est prudent; il s'organise graduellement, secteur par secteur, en particulier dans l'industrie. Puis, à la fin des

(9) Comme nous le verrons dans la deuxième partie, cette date n'est pas fortuite car elle marque l'entrée en vigueur de la ZLEA.

(10) En Argentine, si l'on excepte l'épisode Perón entre 1973 et 1976, les militaires dirigent le pays de 1955 à 1982. Ils chutent après le conflit des Malouines et Raúl Alfonsín devient président de la République en 1983. Au Brésil, un régime militaire prévaut de 1964 à 1985, année où Tancredo Neves devient président. Au Paraguay, le général Stroessner s'empare du pouvoir en 1954 et impose ensuite une dictature jusqu'en 1989, date où il est renversé par le général Andrés Rodríguez qui, une fois confirmé à la tête de l'État par une élection présidentielle, engage son pays sur la voie de la démocratisation. Enfin en Uruguay, une dictature militaire domine la vie politique entre 1976 et 1984 ; Julio Sanguinetti est élu démocratiquement la même année.

(11) "Cette clause n'est pas veine. Elle fut appliquée pour la première fois lors d'une crise politique au Paraguay en avril 1999: la menace émise par les membres les plus importants du MERCOSUR d'en exclure ce pays si les règles de la démocratie n'y étaient pas respectées contribua à résoudre le conflit qui avait pris la forme d'une rébellion militaire." (Schvarzer, 1999 : 82) 
années 1980, l'accession à la présidence de C. Menem en Argentine et de F. Collor de Mello au Brésil marque un changement de cap. Les deux présidents sont favorables à une politique d'ouverture multilatérale. Cependant, les négociations entre le Canada, les États-Unis et le Mexique en vue de signer l' accord l'ALENA (Accord de Libre Échange de l'Amérique du Nord) amènent les deux pays à privilégier la stratégie de régionalisation commerciale. Celle-ci débouche sur la signature du traité d'Asunción en 1991. Le traité prévoit la constitution d'une ZLE à la fin de 1994 et d'une union douanière le $1^{\text {er }}$ janvier 1995 qui doit ensuite évoluer vers un marché commun.

L'objectif économique du MERCOSUR est double. Au niveau interne, il est de créer un espace économique intégré afin de renforcer les économies des pays. Il s'agit d'augmenter le commerce au sein de la région à travers une spécialisation accrue. Le second objectif, qui dépend largement de la réussite du premier, est l'ouverture sur l'extérieur. L'intégration régionale en augmentant la taille des marchés intérieurs de ces membres doit attirer les investissements directs étrangers. À cette double dimension, il s'en ajoute une troisième, à savoir, l'élargissement du MERCOSUR à d'autres (groupes de) pays.

L'accord repose sur une logique "confédérale" qui se différencie des structures fédératives et supranationales européennes. Il n'y a pas une instance publique suprême communautaire pour faciliter l'intégration. Les conflits sont résolus au niveau des quatre présidents, ce qui peut contrarier le processus d'intégration.

L'application du calendrier d'Asunción, rapide jusqu'en 1995, connaît ensuite

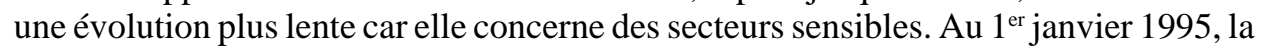
circulation des biens est libre pour $85 \%$ des produits. En retard dans l'échéancier, les membres ont repoussé à 2000 la constitution d'une véritable ZLE. Depuis 1995, la liste des produits taxés est en baisse régulière à l'exception significative du secteur de l'automobile qui bénéficie d'un régime spécial de protection transitoire. Il existe un TEC compris entre 6 et $20 \%$ avec neuf niveaux intermédiaires mais son application est difficile. Une liste d'exceptions recouvre $15 \%$ des produits (12). L'union douanière ne sera pas véritablement réalisée avant 2006.

Bien qu'en retard sur l'échéancierinitial, le processus d'intégration est relativement soutenu. La réussite de l'accord et la volonté politique d'élargissement conduisent à accorder le statut de membre associé (signature d'un traité de libre échange) au Chili (13), en 1996 et à la Bolivie, en 1997.

Néanmoins, le processus reste fragile. En 1999, le MERCOSUR a connu, pour la première fois depuis sa création, une récession économique (-1,3\%). De plus, suite à la perte de compétitivité vis à vis du Brésil (qui a dévalué le real en janvier 1999), l'Argentine a rétabli des protections en contradiction avec les dispositions mercosuriennes. Ces éléments ont conduit, pour la première fois depuis 1986, à une contraction du

(12) L’Argentine, le Brésil et l'Uruguay peuvent maintenir jusqu'au 31 décembre 2000, 300 positions douanières en dehors du TEC. Le Paraguay jouit d'un délai supplémentaire jusqu'au 31 décembre 2005.

(13) Le Chili n'est membre ni du MERCOSUR ni de la CAN et privilégie une intégration régionale bilatérale (voir annexe 1) qui soutienne le projet de ZLEA. 
commerce intra-zone en 1999 (voir infra). Cette crise constitue un test permettant d'éprouver la solidité de l'accord (14).

En conclusion, le MERCOSUR a créé un espace régional entre des pays qui n'avaient guère de liens entre eux (connexions physiques et échanges commerciaux) à cause, notamment, de la présence de régimes militaires autoritaires et nationalistes. Cette situation de départ, combinée à la reprise de la croissance économique dans la région, a permis un processus d'intégration commerciale dynamique. Depuis 1995, l'accord est entré dans une phase de maturation où les intérêts particuliers et les instabilités macroéconomiques rendent les évolutions vers l'intégration plus lentes. De leurs côtés, les membres du Pacte andin (rebaptisé CAN en 1996) ont mis presque 25 ans à constituer une ZLE. Ce succès a créé une dynamique favorable à l'intégration; mais l'approfondissement de celle-ci est soumis à des aléas économiques (15) et politiques.

\section{3. Quelques statistiques descriptives sur les deux accords}

\section{3. 1. Quelques chiffres (annexe 2)}

En tant qu'initiateurs du MERCOSUR et de par leur poids économique (plus de 97\% du PIB de l'accord), l'Argentine et le Brésil constituent le noyau dur du bloc. Mais en raison de ses dimensions (78\% de la population, $71 \%$ de la superficie), le Brésil est le centre névralgique du bloc. Le Paraguay se démarque de ses voisins : le pays est relativement pauvre, souffre d'un enclavement géographique et l'agriculture représente $25 \%$ du PIB (contre $8 \%$ en moyenne pour la région). L'espérance de vie dépasse 70 ans, sauf au Brésil (67 ans).

La CAN est composée de pays plus homogènes au niveau de la population et de la superficie. Ce bloc est plus pauvre que le MERCOSUR (PIB par tête respectifs de $2500 \$$ et $5100 \$$ ), mais on y vit en moyenne deux années de plus. La structure économique de la CAN est relativement plus industrielle (présence du pétrole en Bolivie, en Colombie, en Équateur et au Venezuela). Le taux d'ouverture est de 37\% alors que celui du MERCOSUR, fortement pondéré par le poids de l'Argentine et du Brésil, est de $20 \%$ (égal à celui de l'ALENA [16]).

\section{3. 2. $\operatorname{La} C A N$}

En 1999, le commerce de la CAN dépasse légèrement 80 milliards de dollars. Le Venezuela et la Colombie représentent presque $70 \%$ du commerce de la région (annexe

(14) En 2001, l'économie argentine entre dans une troisième année de récession et la crise prend, depuis cette année, une dimension sociale et politique. Cette crise augmente les incertitudes quant aux évolutions du MERCOSUR.

(15) En 1999, la récession dans la région (- 4,8\%) s'est accompagnée d'une contraction du commerce intra-zone.

(16) À titre de comparaison, en 1998, le taux d'ouverture de l'Union Européenne (UE) est de $50 \%$ et il est de $100 \%$ pour l'Association des États d'Asie du sud-est (ASEAN). 
3). La répartition géographique du commerce de la région est présentée dans l'annexe 4 (17). Les États-Unis sont les premiers partenaires commerciaux de la CAN tant au niveau des exportations (45\%) que des importations (35\%). La proximité géographique des États-Unis peut expliquer cette dépendance, plus forte que celle du MERCOSUR. Ce dernier est un fournisseur non négligeable de la CAN (7\%), alors que ce n' est pas vraiment le cas dans l'autre sens $(2 \%)$.

Entre 1995 et 1999, le commerce intra-zone moyen est légèrement au-dessous de $12 \%$. Il a presque triplé par rapport à la période 1975-1989. Pendant ces 15 années, le commerce intra-zone (graphique 1 [18]) stagne victime, dans les années 1970, du mauvais fonctionnement de l'accord et, dans les années 1980, de la crise de la dette. Au début des années 1990, la reprise de la croissance économique et la réactivation de l'accord ont dynamisé le commerce intra-zone.

L'étude par pays pour la période 1995 et 1999 (annexe 5) met en lumière la dépendance de la Bolivie vis à vis du MERCOSUR. Elle effectue $23 \%$ de ses importations et $16 \%$ de ses exportations avec le MERCOSUR. Cette dépendance commerciale explique sa volonté d'intégrer le MERCOSUR. Par ailleurs, la CAN est un marché important pour les exportations boliviennes (21\% [19]) et colombiennes (18\%).

Nous retenons les points suivants :

- un bloc commercial relativement plus homogène et ouvert que le MERCOSUR.

- un commerce majoritairement orienté vers les États-Unis.

- un commerce intra-zone en nette augmentation depuis le début des années 1990.

- la dépendance commerciale de la Bolivie vis à vis du MERCOSUR.

\section{3. 3. Le MERCOSUR}

En 1999, le commerce du MERCOSUR s'élève à 155 milliards de dollars. L'Argentine et le Brésil représentent 93\% du commerce total de l'accord (voir annexe 3).

Entre 1975 et 1990, le principal partenaire commercial était l'UE, suivie des États-Unis. Le commerce intra-zone se situait au-dessous de 10\%. Puis, à partir du début des années 1990, date de la signature du traité d'Asunción, le commerce au sein du MERCOSUR augmente nettement. En 1999, le commerce intra-zone s'élève à 20\%,

(17) Les calculs sont effectués pour 5 périodes depuis 1975 : 1975-1979, 1980-1984, 1985 1989, 1990-1994 et 1995-1999. Un travail sur des périodes de 5 années se justifie car la répartition par zone du commerce peut connaître des années atypiques. De plus, le découpage correspond à 5 périodes historiques pour la région sud-américaine : la première correspond à l'essoufflement de la politique de substitution aux importations, la seconde marque le début de la crise de la dette, de fortes turbulences macro-économiques caractérisent la troisième période, la quatrième correspond au retour de la croissance économique et à une politique de régionalisation commerciale dynamique ; enfin, la dernière période se caractérise par une phase de maturation du processus d'intégration commerciale.

(18) Nous représentons l'évolution de la part des exportations et des importations au sein de la région qui sont par définition égales aux taxes et aux erreurs de mesure près.

(19) Presque 50\% des exportations boliviennes sont donc destinées au marché sud-américain. 
après s'être situé à 23\% en 1998 (20). Cette évolution suggère que le MERCOSUR a réussi à promouvoir le commerce entre les pays membres.

Une analyse par pays met en évidence une répartition du commerce différente selon les pays. L'Argentine, le Paraguay et l'Uruguay orientent leur commerce majoritairement au sein de la région (autour de $30 \%$ pour le premier et entre 40 et $45 \%$ pour les deux autres). En revanche, le Brésil commerce principalement avec les ÉtatsUnis et l'UE. Le commerce brésilien avec ses partenaires du MERCOSUR a cependant doublé entre 1985-1989 et 1994-1999 pour s'élever à 15\%.

Nous retenons les points suivants:

- un bloc commercial asymétrique dominé par le Brésil.

- une intégration commerciale rapide (le commerce intra-zone est désormais majoritaire) dont la dynamique s'est ralentie depuis la fin des années 1990.

- une dépendance commerciale vis à vis du MERCOSUR plus importante pour l'Argentine, le Paraguay et l'Uruguay que pour le Brésil.

- une plus forte dépendance au commerce international du Paraguay et de l’Uruguay par rapport au Brésil et à l'Argentine.

\section{Graphique 1 - Évolution du commerce (exportations + importations) intra-zone du MERCOSUR et de la CAN entre 1975 et 1999 (en pourcentage du commerce total).}

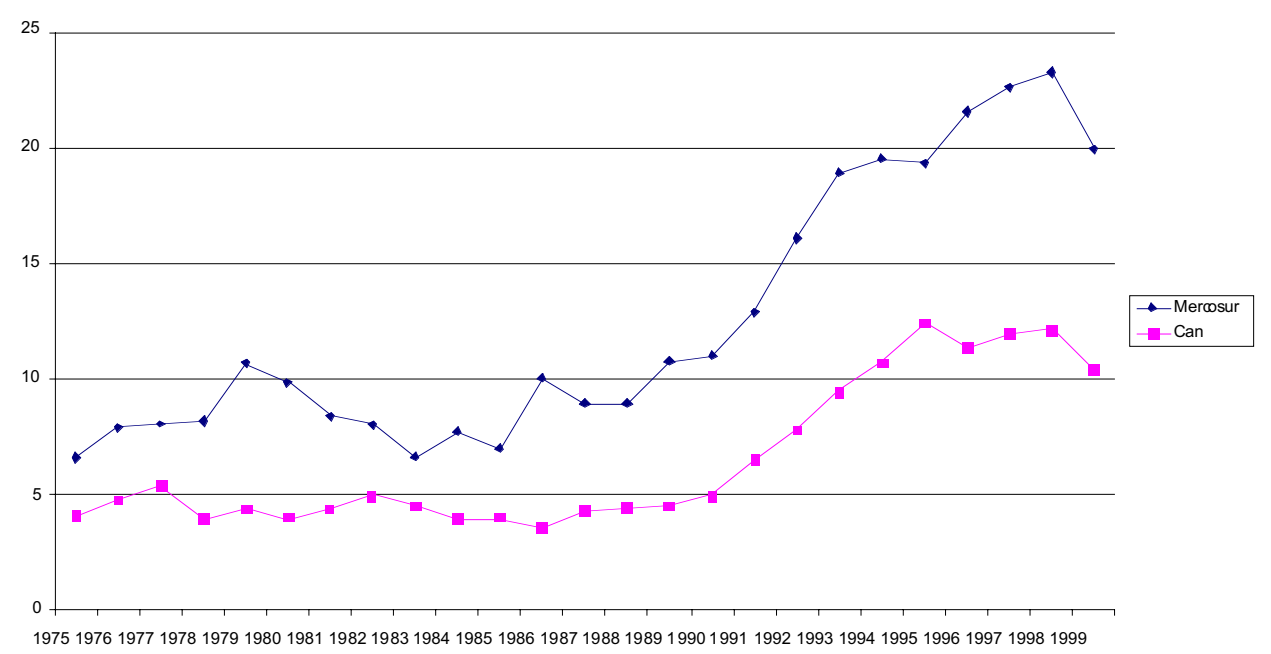

Source : Direction du commerce du FMI.

(20) À titre de comparaison, en 1998, les pays de l'UE effectuent 50\% de leurs échanges entre eux. Ce taux se situe entre 40 et $50 \%$ pour l'ALENA, et dépasse $20 \%$ pour l'ASEAN (Jadresic et al., 1999). 
Pendant la décennie 1990, l'intégration régionale en Amérique du sud, dans le cadre de la CAN et du MERCOSUR, est globalement un succès. 1999, année de récession pour la région, marque une baisse sensible du commerce régional des deux accords. Cette situation permet d'éprouver la solidité des engagements pris en matière de libre échange régional depuis 10 ans.

La ZLEA est prévue pour 2005. Face à cette perspective, les pays membres de la CAN et du MERCOSUR ont entamé des négociations en vue de constituer une ZLE avant l'entrée en vigueur de la ZLEA (21). Nous abordons maintenant les enjeux économiques et politiques attachés au projet de libre commerce en Amérique du sud.

\section{UNEZONE DE LIBREÉCHANGE ENTRE LA CAN ET LE MERCOSUR ?}

D'un point de vue économique, un accord commercial trouve une justification s'il dégage des bénéfices significatifs.

\section{1. Aspects économiques}

L'effet d'un accord commercial peut se décomposer en effets statiques et dynamiques.

\section{1. 1. Effets statiques}

a) Eléments de théorie

L'analyse de Viner (1950) permet d'identifier l'impact contradictoire d'un accord régional sur le bien être. Suite à la constitution d'une ZLE régionale (22) :

- il y a création de commerce lorsqu'un membre abandonne (en partie ou totalement) la production d'un bien pour désormais l'importer d'un autre membre dont le coût de production est relativement moins élevé que lui.

- il y a détournement de commerce lorsqu'un membre remplace l'importation d'un bien d'un pays tiers pour celle d'un autre membre disposant d'un coût de production relativement plus élevé que le pays tiers

L'effet création de commerce, en améliorant l'efficacité allocative des facteurs de production, augmente le bien être de la ZLE, alors que l'effet détournement de commerce le diminue. Cependant, depuis Meade (1955) et Lipsey (1957) on sait qu'il faut réintroduire les effets de consommation qui ont un effet favorable sur le bien être (les consommateurs s'approvisionnent à un coût inférieur après la ZLE) qui peut éventuellement dépasser l'effet initial de détournement constaté en termes de coûts de production. Le gain statique net sera d'autant plus grand que: (i) l'élasticité-prix de demande d'importation (et donc celles de demande et d'offre domestiques) est forte, (ii)

(21) Il faut cependant rester prudent face à une telle hypothèse. L'expérience de l'intégration commerciale dans la région montre que des dispositions prises peuvent être retardées ou non appliquées par la suite.

(22) Pour une présentation graphique de ces effets voir De Melo \& Grether (1997) ou l'annexe 6. 
le tarif douanier initial est élevé et (iii) la différence entre les coûts des pays partenaires et des pays tiers est faible.

\section{b) Éléments d'appréciation des effets d'une ZLE CAN-MERCOSUR}

Le commerce entre la CAN et le MERCOSUR s'élève en 1999 à 4,6 milliards de dollars, soit $2 \%$ du commerce total de la région. Il est structurellement déficitaire pour la CAN et en 1999 ce déficit s'élève à 700 millions de dollars.

La structure du commerce bilatérale (23) (graphique 2) est excédentaire pour le CAN dans les branches des minerais et de l'énergie. Le commerce de toutes les autres branches est excédentaire pour le MERCOSUR, en particulier dans la branche de la mécanique électrique. Le commerce entre les deux régions est concentré dans les branches de l'énergie et de la mécanique électrique qui représentent (en moyenne entre 1996 et 1998) 44\% du commerce bilatéral total.

\section{Graphique 2 - Décomposition moyenne du commerce entre la CAN ${ }^{a}$ et le MERCOSUR ${ }^{\mathrm{b}}$ entre 1996 et 1998 (en millions de dollars).}

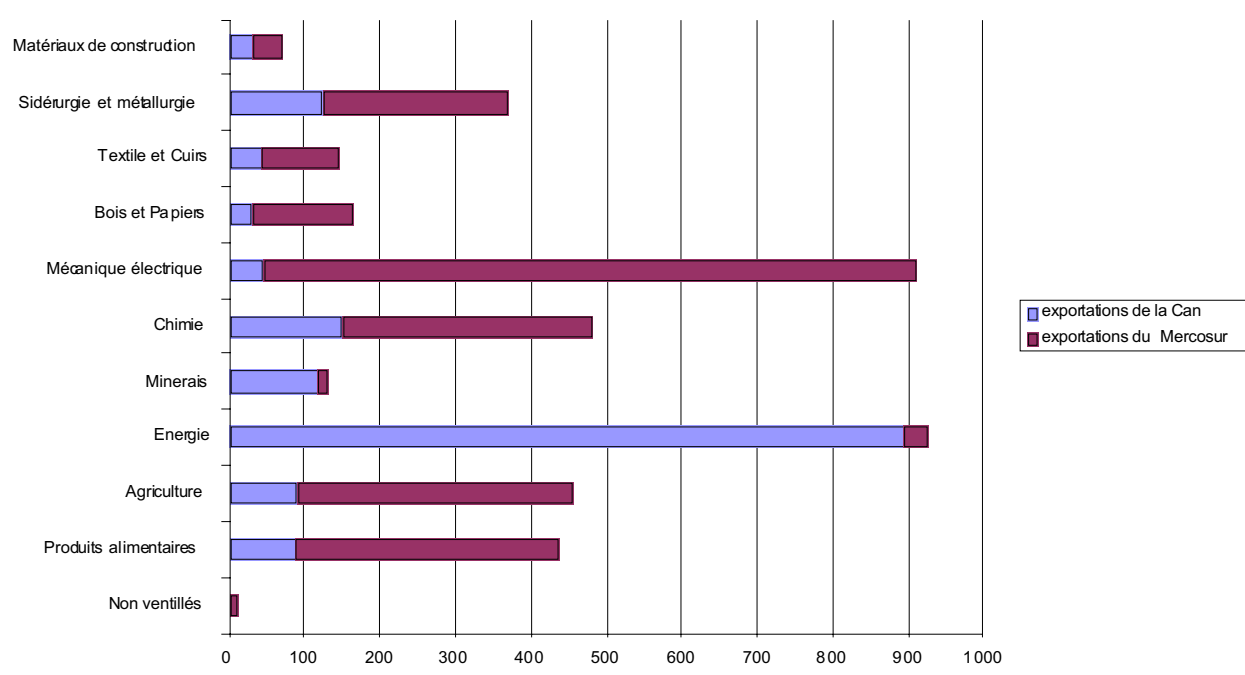

${ }^{\text {a }}$ sans la Bolivie.

${ }^{\mathrm{b}}$ sans le Paraguay et l'Uruguay.

Source : Chelem, 2000.

(23) Les données décomposant le commerce par branche proviennent de Chelem, 2000. Cette base de données, qui servira à calculer les avantages comparatifs révélés (voir infra), ne comprend pas la Bolivie, le Paraguay et l'Uruguay. Nous acceptons cette restriction compte tenu du fait que la part du commerce de ces trois pays dans le commerce total de leurs accords respectifs est relativement faible: respectivement $4 \%, 3 \%$ et $4 \%$ (annexe 3 ). 
Une évaluation statique ex-ante d'un éventuel accord CAN-MERCOSUR est par définition impossible. Cependant, en se basant sur les résultats de la littérature sur les accords régionaux, on peut penser que les effets de détournement pourraient dominer les effets de création de commerce. D'abord, il semble qu'il y ait “consensus au sujet du fait que les accords régionaux (...) entre pays en développement (comme ceux de la CAN et du MERCOSUR) ont eu peu d'effets à cause des difficultés de mise en œuvre liées particulièrement aux manques de mécanismes compensatoires" (De Melo \& Grether, 1997 : 713). Par ailleurs, les évaluations a posteriori, de l'ALENA ou du Marché Unique n'ont pas mis en évidence des gains qui dépassent significativement le degré d'incertitude attaché aux méthodes et aux statistiques (Winters, 1997 in : Fouquin \& Siroên, 1998). Même en ce qui concerne le MERCOSUR, où l'explosion des échanges bilatéraux pouvait laisser croire à la prédominance des effets de création, les résultats sont ambigus (Yeats, 1997 ; Miotti et al., 1998).

À partir des trois conditions déterminant l'ampleur de l'effet net sur le bien être d'une ZLE (voir supra ou annexe 6), on peut penser que l'impact d'un accord entre la CAN et le MERCOSUR aurait un signe ambigu.

D'abord, il est reconnu que l'élasticité de la demande d'importation par rapport au prix est relativement faible dans les pays en développement (De Melo \& Grether, 1997). Cependant, la libéralisation des économies (en particulier le marché du travail) sud américaines, depuis la deuxième moitié de la décennie 1980, a dû entraîner une plus grande élasticité de l'offre domestique (et donc de la demande d'importation).

Ensuite, le niveau des barrières tarifaires des pays sud-américains (Tab. 1), qui a fortement baissé dans le cadre de la politique de libéralisation unilatérale et multilatérale (24), serait susceptible de limiter les effets positifs d'une ZLEet ceci de deux manières : en réduisant les effets de création de commerce et en limitant l'impact positif de consommation dans le cas du détournement de commerce.

Tableau 1 : Données de tarifs douaniers en 1999.

CAN (25)

\begin{tabular}{|l|ccllll|}
\hline & Bolivie & Colombie & Équateur & Pérou & Venezuela & CAN \\
\hline Tarif moyen (\%) & 9,7 & 11,6 & 11,5 & 13,7 & 12 & 11,7 \\
Intervalle (\%) & $2-10$ & $0-35$ & $0-99$ & $0-68$ & $0-35$ & $0-99$ \\
Écart type & 1,3 & 6,3 & 7,8 & 4,8 & 6,1 & $\mathrm{nd}^{\mathrm{a}}$ \\
Mode (\%) & 10 & 5 & 5 & 12 & 5 & $\mathrm{nd}$ \\
\hline
\end{tabular}

${ }^{a}$ nd : non disponible*

(24) En 1985, le tarif douanier moyen de la région sud-américaine dépassait 50\% (Edwards, 1995).

(25) Pour la CAN, qui constitue une ZLE sans exception, le tarif douanier moyen de 11,7\% correspond au TEC de la région. Pour le MERCOSUR, le taux de 12,9\% ne correspond pas au TEC mais y tend au fur et à mesure que tous les tarifs au sein de la région sont ramenés à zéro. 
MERCOSUR

\begin{tabular}{|l|clccc|}
\hline & Argentine & Brésil & Paraguay & Uruguay & MERCOSUR \\
\hline Tarif moyen (\%) & 13,5 & 14,3 & 11,4 & 12,3 & 12,9 \\
Intervalle (\%) & $0-33$ & $0-35$ & $0-30$ & $0-23$ & $0-35$ \\
Écart type & 6,6 & 7 & 6,8 & 7 & nd \\
Mode (\%) & 5 & 19 & 5 & 5 & nd \\
\hline
\end{tabular}

Source : Banque Inter-Américaine de Développement.

La condition (iii) pourrait, cependant, compenser ce résultat négatif. Une quantification de l'effet par les coûts de production passe par une comparaison microéconomique par branche entre la CAN et le MERCOSUR et les pays tiers (en particulier les États-Unis et l'UE) qui dépasse le cadre cet article. Néanmoins, nous pouvons avoir une idée de cet effet à partir de la proposition selon laquelle les créations de commerce seraient maximisées dans le cas d'un commerce inter-branche basé sur des avantages comparatifs (26) très différents (de la Torre \& Kelly, 1992). Dans un contexte international de baisse général des tarifs, le fait, qu' un pays formant une ZLE avec un autre partenaire dispose d'un avantage comparatif élevé vis à vis de celui-ci, peut signifier que les différences de coûts de production avec le reste du monde seraient réduites (ou en tout cas moindre par rapport au cas où il n'y aurait pas d'avantages comparatifs élevés). Quels sont les avantages comparatifs, entre la CAN et le MERCOSUR ? Existe-t'il des avantages comparatifs très différents ?

En théorie, le calcul des avantages comparatifs découle d'une comparaison des dotations factorielles (capital-travail), mais dans le pratique ce type de calcul est difficile pour les pays en développement (manque de données). Nous approximons l'avantage comparatif à partir d'une méthode du CEPII, dite de l'avantage comparatif révélé (ACR) se basant sur la contribution pour chaque branche au solde commercial (voir annexe 7 pour le calcul et la signification de l'indicateur). Nous proposons dans le tableau 2, le calcul de cet indicateur entre la CAN et le MERCOSUR en moyenne pour la période 1996-1998. Le calcul sur une période de trois années se justifie afin de limiter les effets des changements temporaires de balance commerciale dus, par exemple, à un essor de la consommation ou une variation non soutenable du taux de change.

(26) La théorie des avantages comparatifs constitue depuis Ricardo le socle de la théorie internationale du commerce. Tout pays aura avantage à se spécialiser (et donc à commercer) dans la production où il a un avantage absolu ; s'il n'en a pas, il aura quand même intérêt à se spécialiser dans la production où il dispose de l'avantage relatif (comparatif) le moins défavorable. 
Tableau 2 - Avantages comparatifs révélés moyens de la $\mathrm{CAN}^{\mathrm{a}}$ vis à vis du MERCOSUR $^{\mathrm{b}}$ entre 1996 et 1998 (en PIB de la CAN/1000). (27)

\begin{tabular}{|l|c|}
\hline \multicolumn{1}{|c|}{ Branches } & ACR \\
\hline Matériaux de construction & 0,04 \\
Sidérurgie et métallurgie & $-0,17$ \\
Textiles et cuirs & $-0,11$ \\
Bois et papiers & $-0,26$ \\
Mécanique électrique & $-2,38$ \\
Chimie & $-0,30$ \\
Minerais & 0,51 \\
Énergie & 4,03 \\
Agriculture & $-0,69$ \\
Produits alimentaires & $-0,64$ \\
Non ventilés & $-0,03$ \\
\hline
\end{tabular}

${ }^{\text {a }}$ sans la Bolivie.

${ }^{\mathrm{b}}$ sans le Paraguay et l'Uruguay.

Source : Chelem, 2000. Calculs de l'auteur.

Les deux régions disposent chacune d'un avantage comparatif clair : la filière énergie (qui comprend en particulier le pétrole) pour la CAN et la mécanique électrique pour le MERCOSUR. Un accord de libre échange en Amérique du sud, en exploitant ces avantages comparatifs (via la spécialisation), produirait des effets positifs susceptible de compenser les effets de détournement de commerce. De plus, ce résultat n'est pas neutre du point de vue de la politique économique : il est plus facile de libéraliser le commerce s'il existe un avantage absolu clair pour chaque économie (moindre pression des catégories perdantes et plus grande marge de manœuvre des pouvoirs publics pour les indemniser et pour faciliter la reconversion).

Au total, un accord de libre échange entre la CAN et le MERCOSUR aurait des effets statiques contradictoires en terme de bien être pour la région qui pourraient être finalement marginaux. Cette proposition découle d'une analyse ex-ante et reste donc fragile. De plus, il convient de ne pas surestimer ces effets statiques car il est généralement admis que des effets dynamiques les dominent à long terme (Bourguinat, 1992).

(27) L'ACR du MERCOSUR vis à vis de la CAN donne évidemment un même ordre de classement mais inversé. Une valeur positive dans le tableau 2 signifie un avantage comparatif de la CAN, une valeur négative signifie un désavantage comparatif autrement dit, un avantage comparatif du MERCOSUR vis à vis de la CAN. 


\section{1. 2. Effets dynamiques}

Ces effets, contrairement aux précédents, provoquent un changement économique durable. Benaroya (1995) en retient trois principaux :

-la meilleure diffusion technologique et les économies d'échelle dans un marché local devenu plus large qui permettent, dans un deuxième temps, de mieux affronter la compétition internationale.

- une concurrence interne renforcée, qui incite à une meilleure efficacité productive.

- un climat plus favorable pour les investissements directs étrangers. Cet effet est d'autant plus élevé que le marché régional est grand. Si une dynamique favorable est enclenchée, il apparaît un phénomène auto-entretenu car les investisseurs ont tendance à se grouper ensemble (Ethier, 1998). L'Amérique du sud est restée relativement fermée pendant plusieurs décennies. La politique d'ouverture unilatérale des années 1980 a attiré les investissements directs étrangers, et la régionalisation, en particulier dans le cadre du MERCOSUR (28), a permis une forte hausse de ce type d'investissement. La CAN a relativement moins bénéficié de cet effet. On peut penser que la réunion de ces deux zones, en augmentant la taille du marché, est susceptible d' augmenter la dynamique de flux des investissements directs étrangers dans la région, en particulier pour les pays andins.

La littérature propose peu de travaux proposant une estimation des effets dynamiques. Des auteurs comme Mistry (1996), Fernandez (1997) proposent de les modéliser mais les tests empiriques de ces modèles sont encore déficients (Winters, 1997 in : Delvin \& Ffrench-Davis, 1999). On peut penser que ces effets devraient être élevés compte tenu du fait que la région est restée relativement fermée vis à vis du reste du monde entre 1930 et 1980 et que l'intégration régionale a lieu dans une perspective de régionalisme ouvert, c'est à dire dans le cadre d'une ouverture commerciale unilatérale des pays membres.

Si les effets statiques d'un accord entre la CAN et le MERCOSUR sont ambigus, les effets dynamiques pourraient être positifs. L'effet global de cette ZLE pourrait être positif mais cette proposition (découlant d'une analyse ex-ante), compte tenu de son manque de robustesse, ne peut expliquer et justifier le projet de libre commerce CANMERCOSUR. En fait, les raisons profondes de ce projet sans doute plus dans des considérations stratégiques. En effet, si les deux blocs commerciaux cherchent à s'unir, c'est aussi pour constituer un bloc suffisamment solide pour pouvoir peser dans les négociations sur la future ZLEA.

\section{2. Aspects stratégiques}

Le projet de ZLEA a profondément modifié la dynamique d'intégration commerciale en Amérique du sud.

(28) Entre 1984 et 1989, le MERCOSUR recevait 2\% des flux mondiaux d'investissements directs étrangers, en 1997, 6,6\% des flux globaux lui sont destinés (Giordano \& Santiso, 2000). 
L'idée de libre échange continental est né, aux États-Unis, au début des années 1990 pendant les négociations de l'ALENA.

“Il apparaît aujourd'hui beaucoup plus clairement que ce n'était le cas à l'époque, qu'en négociant avec le Canada et le Mexique un accord de libre échange, les États-Unis avaient des ambitions beaucoup plus grandes que celles que leurs deux partenaires leur imputaient." (Deblock \& Brunelle, 1999 : 40).

En effet, au-delà de la libéralisation du commerce et de l'investissement, l'accord de l'ALENA devait prendre la valeur de modèle dans les négociations commerciales subséquentes (29).

En Amérique du sud le projet est accueilli de manière ambiguë. Tous les pays, bien qu'à des degrés divers, souhaitent accéder au marché nord-américain et attirer des investisseurs. Mais en même temps, la vision nord-américaine d'imposer une base de discussion dite "OMC plus" (à l'image de l'ALENA) suscite des craintes (Dabène, 2000). Les États-Unis, dont le PIB représente $75 \%$ du continent, veulent imposer un traité sur des domaines nouveaux, les new issues (en particulier, la non-discrimination lors de la passation des marchés publics et la libéralisation dans le domaine de la propriété intellectuelle) et d'autres très nouveaux, les brand new issues (en particulier, le respect de certaines normes environnementales, le droit des investisseurs étrangers vis à vis des États nationaux et le droit au travail, notamment, l'interdiction du travail des enfants). Les pays d'Amérique du sud, qui ont déjà du mal à appliquer les dispositions de l'OMC, s'opposent à une telle vision, dont l'application pourrait pénaliser leurs économies. Par ailleurs, l'Amérique du sud dénonce la position des États-Unis qui consiste à chercher à protéger certains produits agricoles et le secteur de l'acier. Il est donc important pour la région, et en particulier pour le Brésil (économie moins libéralisée que les autres grands pays de la région), de former un bloc uni et cohérent pour espérer peser dans les négociations. Face à la vision nord-américaine d'un libre échange pan continental sur la base d'un élargissement de l'ALENA, la CAN et le MERCOSUR insistent d'abord sur la nécessaire consolidation du processus d'intégration commerciale sud-américain. Le Brésil est le principal acteur de cette stratégie.

Le sommet de Miami, en décembre 1994, qui a réuni les trente-quatre pays du continent (à l'exclusion de Cuba), aboutit à la signature d'un document final prévoyant l'entrée en vigueur de la ZLEA en 2005 (30), mais restant assez vague quant au moyen pour y parvenir. Ce manque de clarté va profiter au Brésil qui va pouvoir infléchir, dans un sens conforme à ses intérêts, la stratégie de ses voisins, d'abord dans le cadre du MERCOSUR puis en direction de la CAN.

(29) Baldwin (1997) parle d'effet domino.

(30) Si le sommet de Miami fait apparaître des divergences concernant la libéralisation commerciale, il se caractérise aussi par une remarquable convergence sur les thèmes de la préservation et de l'approfondissement de la démocratie, la lutte contre la pauvreté et la lutte contre le crime organisé et la corruption. 
En 1995, même sice n'est pas officiel, le processus d'intégration du MERCOSUR passe d'une logique d'élargissement (31)à une logique d' approfondissement de l' accord en consolidant l'union douanière, entrée en vigueur en 1995, et en avançant vers le marché commun (Dabène, 2000). Ce changement de stratégie n'empêche pas la signature des traités accordant un statut de membre associé au Chili en 1996 et à la Bolivie en 1997, interlocuteurs de la première heure pour l'élargissement. En revanche, les négociations bilatérales engagées avec la Colombie, l'Équateur, le Mexique, le Pérou et le Venezuela échouent pendant la même période.

La CAN, suite au sommet de Miami, adopte une position un peu différente. Comme le MERCOSUR, elle redoute d'être diluée dans la ZLEA. Elle cherche donc à consolider l'accord existant. Mais, contrairement à son voisin du sud, dont le poids géostratégique est évident, cette orientation n'est pas suffisante pour constituer un bloc commercial susceptible de rivaliser avec les États-Unis. Ainsi, les andins mènent une double stratégie d'approfondissement et d'élargissement. Ils lancent à cet effet une initiative destinée à réunir dans un accord commercial la CAN et le MERCOSUR. Le Brésil, conscient de son rôle de première puissance politique et économique dans la région, accueille favorablement l'idée d'un accord régional en Amérique du sud. En 1998, sous son impulsion, le MERCOSUR entame les discussions que sollicitait la CAN ; elles échouent en mars 1999, en grande partie à cause des réticences argentines (32). Le Brésil, prend alors l'initiative de négociations séparées avec les andins ce qui obligent les autres membres du MERCOSUR à emboîter le pas. En mars 2001, la CAN signe avec le Brésil puis l'Argentine un accord de libre échange et le Paraguay et l'Uruguay doivent normalement suivre. L'objectif est que ces accords prennent une dimension multilatérale en 2002, ce qui inaugurerait la ZLE entre la CAN et le MERCOSUR.

\section{CONCLUSION}

Depuis les difficultés dans les négociations du GATT et le ralliement spectaculaire des États-Unis aux thèses régionalistes, les accords de commerce régionaux se sont multipliés entre les pays développés comme parmi les pays en développement. La création du MERCOSUR et la relance de la CAN sont les deux exemples les plus remarquables en Amérique du sud de cette deuxième vague de régionalisme, après celle des années soixante.

Après avoir signé l'ALENA, les États-Unis sont aujourd'hui à l'origine du projet de libre échange pan continental (la ZLEA). Face à cette perspective, les pays de la CAN et le MERCOSUR ont prévu la mise en place, trois ans avant la ZLEA, d'une ZLE. Audelà des arguments économiques, il semble, qu'à travers ce projet, l'Amérique du sud

(31) Comme nous l'avons mentionné, le traité d'Asunción stipule que le MERCOSUR est ouvert aux autres pays.

(32) L'Argentine, pour plusieurs raisons (améliorer la crédibilité de son système monétaire de caisse de convertibilité proche de la dollarisation, contrer les tentations d'hégémonie régionale de son voisin brésilien, légitimer son statut de partenaire privilégié au sein de l'OTAN), est favorable à un rapprochement rapide et profond avec les États-Unis. Mais elle ne peut ni imposer son point de vue aux autorités brésiliennes, ni faire cavalier seul car le Brésil est son principal partenaire commercial. 
(et en premier chef le Brésil) cherche à constituer un bloc commercial susceptible de peser (en particulier face aux États-Unis) dans les négociations portant sur la future ZLEA. Le troisième sommet des Amériques à Québec en avril 2001, dominé par un clivage continental, semble avoir confirmé ce rapport de force. D'un côté, le Canada et le Mexique (membres de l'ALENA), l'Amérique centrale (région déjà de fait dans l'orbite des États-Unis) et le Chili ont soutenu la vision nord-américaine d'un accord de type "OMC plus". De l'autre, les membres de la CAN (surtout à travers le discours du président vénézuélien Hugo Chavez) et du MERCOSUR se sont rangés derrière la vision critique du Brésil. Pour l'heure, ce groupe s'efforce manifestement de retarder autant que possible les échéances en attendant que les États-Unis affichent clairement leurs propositions sur le dossier sensible des produits agricoles.

Malgré ce clivage, la coexistence, après 2005, de deux ZLE, l'une continentale, l'autre entre la CAN et le MERCOSUR, est une hypothèse envisageable. À plus long terme, cette organisation régionale du commerce est susceptible d'influencer l'ordre monétaire de la région, en particulier, à travers un choix entre la dollarisation (Berg \& Borensztein, 2000 ; Dempère \& Quenan, 2000) et l'union monétaire entre les pays de la CAN et du MERCOSUR (Eichengreen, 1999 ; Benassy-Quéré \& Coeuré, 2000 ; García Herrero \& Glöckler, 2001). En 2001, plusieurs éléments laissent penser que l'Amérique du sud pourrait adopter le dollar US : manque de crédibilité des monnaies nationales, économies en partie dollarisées (33), dépendance commerciale vis à vis des États-Unis, adoption officielle du dollar US comme monnaie nationale en Équateur depuis 2000. De plus, si l'accord de la ZLEA œuvre, par la suite, pour une intégration de type marché commun à l'européenne, cela constituerait, à terme, un argument presque définitif pour l'adoption généralisée du dollar sur le continent. Cependant, si la ZLEA se limite à une simple zone de libre commerce (comme c'est actuellement le cas au sein de l'ALENA), l'intégration commerciale sud-américaine pourrait constituer un premier jalon vers une coopération des politiques économiques entre pays membres pouvant déboucher, à terme, vers une union monétaire de la région.

\section{Références citées}

BALDWIN, R., 1997 - The Causes of Regionalism. The World Economy, vol. 20, n 7 :

BENAROYA, F., 1995 - Que penser des accords régionaux ? Économie Internationale, $\mathrm{n}^{\circ} 63$. BENASSY-QUERE, A. \& COEURE, B., 2000 - L'avenir des "petites" monnaies, solutions régionales contre solution en coin. Revue d'Économie Politique, vol 110, $\mathrm{n}^{\circ} 3$.

BERG, A. \& BORENSZTEIN, E., 2000 - The Pros and Cons of Full Dollarization. IMF Working Paper, $\mathrm{n}^{\circ} 50$.

BOURGUINAT, H., 1992 - L'émergence contemporaine des zones et blocs régionaux; Mucchielli et Célimène Eds.

DABÈNE, O., 2000 - Le Mercosur et la Zone de libre-échange des Amériques : vers la convergence ? In : Amérique latine 2000 (Couffignal eds.); Peris : La documentation Française.

(33) Sauf au Brésil, en Colombie et au Venezuela. 
DEBLOCK, C. \& BRUNELLE, D., 2000 - Le projet de Zone de Libre-échange des Amériques, un régionalisme en trois dimensions. In : Amérique latine 2000 (Couffignal eds.) ; Peris : La documentation Française.

DELVIN, R. \& FFRENCH-DAVIS, R., 1999 - Towards an Evaluation of Regional Integration in Latin America in the 1990s. The World Economy, vol. 22, $\mathrm{n}^{\circ} 2$.

DEMPERE, P. \& QUENAN, C., 2000 - Les débats sur la dollarisation : un état des lieux. In : Amérique latine 2000 (Couffignal eds.) ; Peris : La documentation Française.

EDWARDS, S., 1995 - Crisis and Reform in Latin America : From Despair to Hope, Oxford University Press.

EICHENGREEN, B., 1999 - Does Mercosur need a Single Currency ? NBER Working Paper, $\mathrm{n}^{\circ} 6821$.

ETHIER, W., 1998 - The New Regionalism. The Economic Journal, $\mathrm{n}^{\circ} 108$.

FERNANDEZ, R., 1997 - Returns to Regionalism : An Evaluation of Non-Traditional Gains from RTA's; New York: International Trade Division, World Bank and New York University.

FOUQUIN, M. \& SIROEN, J.-M., 1998 - Régionalisme et multilatéralisme sont-ils antinomiques? Économie Internationale, $\mathrm{n}^{\circ} 78$.

GARCÍA HERRERO, A. \& GLOCKLER, G., 2001 - L'Amérique latine face à la mondialisation : union monétaire ou dollarisation. Problèmes d'Amérique latine, $\mathrm{n}^{\circ} 41$.

GIORDANO, P. \& SANTISO, J., 2000 - La course aux Amériques : les stratégies des investisseurs européens dans le Mercosur. Problèmes d'Amérique latine, $\mathrm{n}^{\circ} 39$.

JADRESIC, E., MASSON, P. \& MAURO, P., 1999 - Exchange Rate Regimes of Developing Countries : Global Context and Individual Choices. FMI, mimeo.

LIPSEY, R., 1957 - The Theory of Customs Union : Trade Diversion and Welfare. Economica, vol. 24.

MEADE, J., 1955 - The Theory of Customs Unions ; Amsterdam, North Holland.

MELO De, J., MONTENEGRO, C. \& PANAGARIYA, A., 1992 - Regional Integration, Old and New. Policy Research Working Papers, $\mathrm{n}^{\circ}$ 985, World Bank.

MELO De, J. \& GRETHER, J.-M., 1997 - Commerce International, Théories et applications ; De Boeck Université.

MIOTTI, L., NICOLAS, F. \& QUENAN, C., 1996 - De la crise de la dette à "l'effet tequila" performances comparées des économies latino-américaines. Problèmes d'Amérique latine, $\mathrm{n}^{\circ} 21$.

MIOTTI, L., QUENAN. C. \& WINOGRAD, C., 1998 - Spécialisation internationale et intégration régionale : l'Argentine et le Mercosur. Économie Internationale, $\mathrm{n}^{\circ} 78$.

MISTRY, P., 1996 - Regional Integration Arrangements in Economic Development; The Hague : FONDAD.

NOGUES, J. \& QUINTANILLA, R., 1993 - Latin America's Integration and the Multilateral Trading System. In : New Dimensions in Regional Integration (De Melo \& Panagariya eds) ; Cambridge University Press.

PANAGARIYA, A., 1996 - The Free Trade Area of the Americas : Good for Lation America?. The World Economy, vol. 19, $\mathrm{n}^{\circ} 5$.

SALGADO, G., 1998 - El Grupo Andino de hoy, Eslabón hacia la integración de sudamérica ; Quito : Universidad Andina Simon Bolivar, Corporación Editora Nacional.

SCHVARZER, J., 1999 - Le Mercosur, du succès à la crise : le poids du Brésil. Problèmes d'Amérique latine, $\mathrm{n}^{\circ} 33$.

TORRE de la, A. \& KELLY, M., 1992 - Regional Trade Arrangements. IMF Occasional Paper, $n^{\circ} 93$.

VINER, J., 1950 - The Customs Unions Issues ; New York : Carnegie Endowment for International Peace. 
WINTERS, A., 1997 - Assessing Regional Integration Arrangements ; Washington D.C : International Trade Division, World Bank.

YEATS, A., 1997 - Does Mercosur's Trade Performance Raise Concerns About the Effects of Regional Trade Arrangements ? Policy Research Working Paper, $\mathrm{n}^{\circ} 1722$; World Bank. 


\section{ANNEXE 1}

Principaux accords commerciaux sur le continent américain ${ }^{\mathrm{a}}$

\begin{tabular}{|c|c|}
\hline Accords & Commentaires \\
\hline $\begin{array}{l}\text { CAN (Bolivie, Colombie, Équateur, Pérou, } \\
\text { Venezuela) }\end{array}$ & $\begin{array}{l}\text { Signature en } 1969 \\
\text { Réactivation en } 1989\end{array}$ \\
\hline $\begin{array}{l}\text { CARICOM (Marché Commun d'Amérique } \\
\text { Central ; Costa Rica, El Salvador, Guatemala, } \\
\text { Honduras, Nicaragua) }\end{array}$ & $\begin{array}{l}\text { Signature en } 1973 \\
\text { Réactivation en } 1990 \\
\text { Favorable à la vision nord- } \\
\text { américaine de la ZLEA }\end{array}$ \\
\hline $\begin{array}{l}\text { MERCOSUR (Argentine, Brésil, Paraguay, } \\
\text { Uruguay) }\end{array}$ & $\begin{array}{l}\text { Signature en } 1991 \\
\text { Deuxième volume de } \\
\text { commerce du continent : } 155 \\
\text { milliards de dollars. }\end{array}$ \\
\hline ALENA (Canada, États-Unis, Mexique) & $\begin{array}{l}\text { Signature } 1993 \\
\text { Premier volume de commerce } \\
\text { du continent : } 2100 \text { milliards } \\
\text { de dollars. }\end{array}$ \\
\hline G3 (Colombie, Mexique, Venezuela) & Signature 1994 \\
\hline Chili - Mexique & Signature 1991 \\
\hline Chili - Colombie & Signature 1993 \\
\hline Chili - Venezuela & Signature 1993 \\
\hline Chili - Costa Rica & Signature 1994 \\
\hline Chili - Équateur & Signature 1994 \\
\hline Chili - MERCOSUR & Signature 1996 \\
\hline Chili - Canada & Signature 1997 \\
\hline Bolivie - MERCOSUR & Signature 1997 \\
\hline Mexique - Nicaragua & Signature 1998 \\
\hline
\end{tabular}

${ }^{a}$ Hors ALADI (ex-ALALE) 


\section{Principaux accords en discussion}

Mercosur - Pacte andin

Mercosur - Union Européenne

Chili - Union Europénne

Mexique - Union Européenne

Mexique - El Salvador, Guatemala, Honduras

Mexique - Équateur

Mexique - Pérou

Mexique - Panama

ZLEA

Source : Devlin \& Ffrench-Davis (1999)

\section{ANNEXE 2}

Indicateurs géographiques, démographiques et sociaux en 1998

\begin{tabular}{|l|ccccc|}
\cline { 2 - 6 } \multicolumn{1}{l|}{} & $\begin{array}{c}\text { Superficie } \\
\text { (milliers de } \\
\left.\mathrm{km}^{2}\right)\end{array}$ & $\begin{array}{c}\text { Population } \\
\text { (milliers) }\end{array}$ & $\begin{array}{c}\text { Densité } \\
\text { population } \\
\text { (habitants } \\
\left./ \mathrm{km}^{2}\right)\end{array}$ & $\begin{array}{c}\text { Taux } \\
\text { d'urba- } \\
\text { sation } \\
(\%)\end{array}$ & $\begin{array}{c}\text { Espérance } \\
\text { de vie } \\
\text { (années) }\end{array}$ \\
\hline CAN & $\mathbf{4 7 1 8}$ & $\mathbf{1 0 8 9 7 2}$ & $\mathbf{2 3}$ & $\mathbf{7 4}$ & $\mathbf{7 0}$ \\
Bolivie & 1098 & 7949 & 7 & 61 & 62 \\
Colombie & 1138 & 40804 & 36 & 73 & 70 \\
Équateur & 283 & 12175 & 43 & 63 & 70 \\
Pérou & 1285 & 24801 & 19 & 72 & 69 \\
Venezuela & 912 & 23242 & 25 & 86 & 73 \\
\hline MERCOSUR & $\mathbf{1 1 9 1 2}$ & $\mathbf{2 1 0 5 0 6}$ & $\mathbf{1 8}$ & $\mathbf{8 1}$ & $\mathbf{6 8}$ \\
Argentine & 2780 & 36125 & 13 & 89 & 73 \\
Brésil & 8547 & 165873 & 19 & 80 & 67 \\
Paraguay & 406 & 5219 & 13 & 55 & 70 \\
Uruguay & 177 & 3289 & 19 & 91 & 74 \\
\hline États-Unis & 9364 & 270300 & 29 & 77 & 77 \\
\hline & & & & & \\
\hline
\end{tabular}




\section{Indicateurs économiques en 1998}

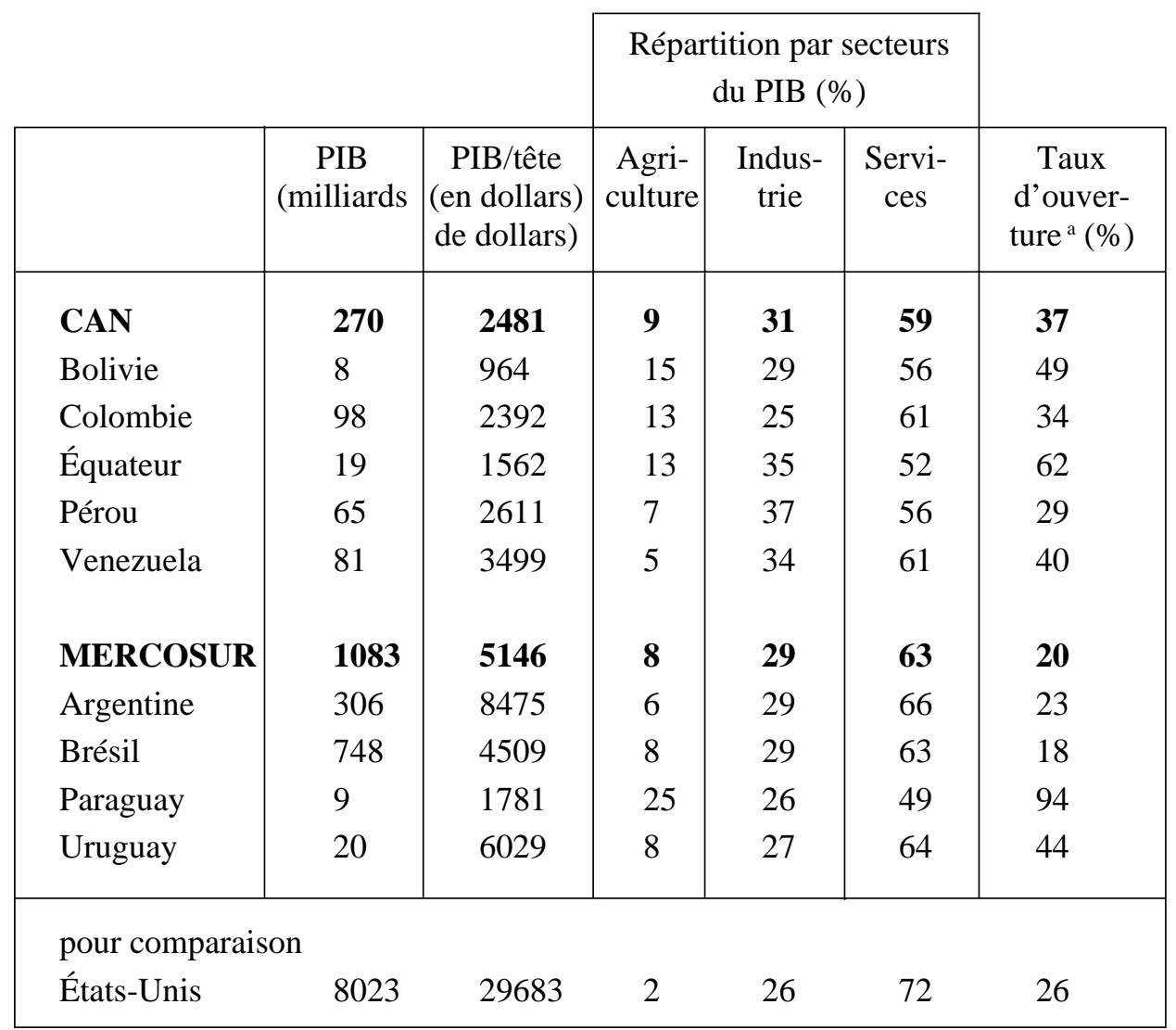

Source: Banque Mondiale.

${ }^{a}$ Exportations plus importations sur PIB 


\section{ANNEXE 3}

Poids relatifs du commerce des différents pays dans le commerce de la CAN et du MERCOSUR entre 1995 et 1999 (en pourcentage total du commerce)

\section{CAN}

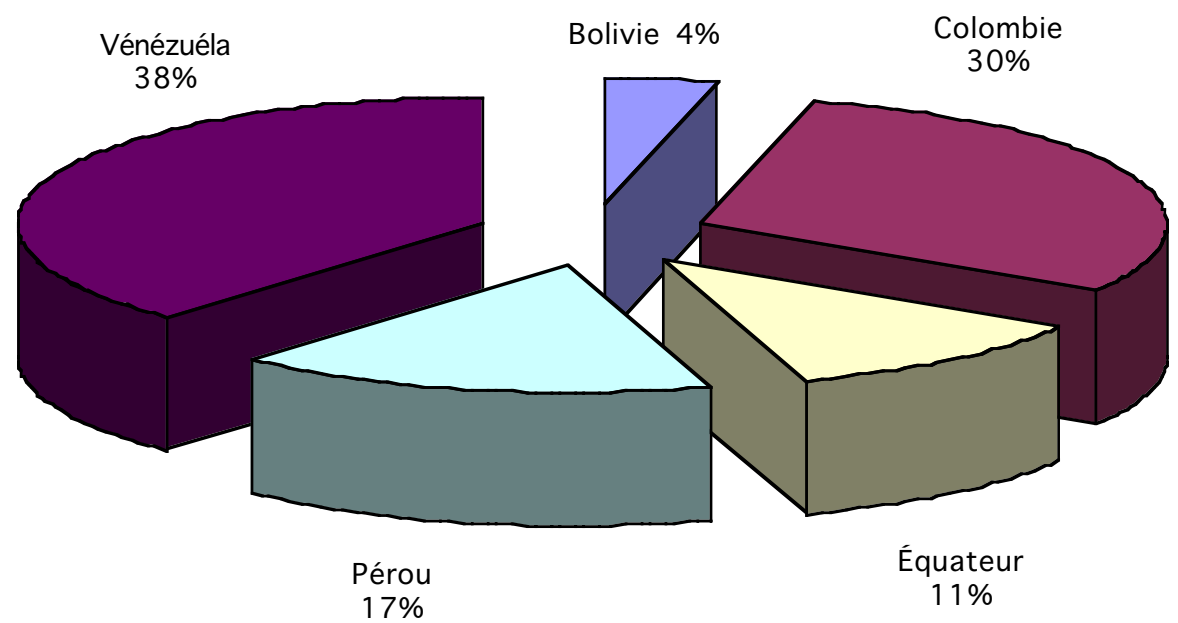

\section{MERCOSUR}

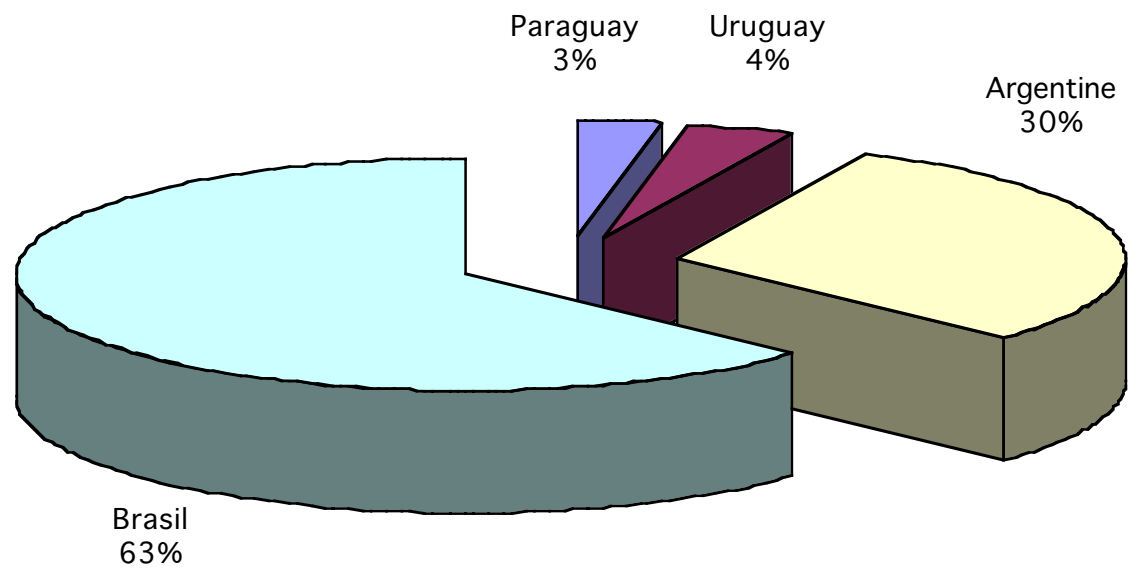

Source : Direction du Commerce du FMI. 


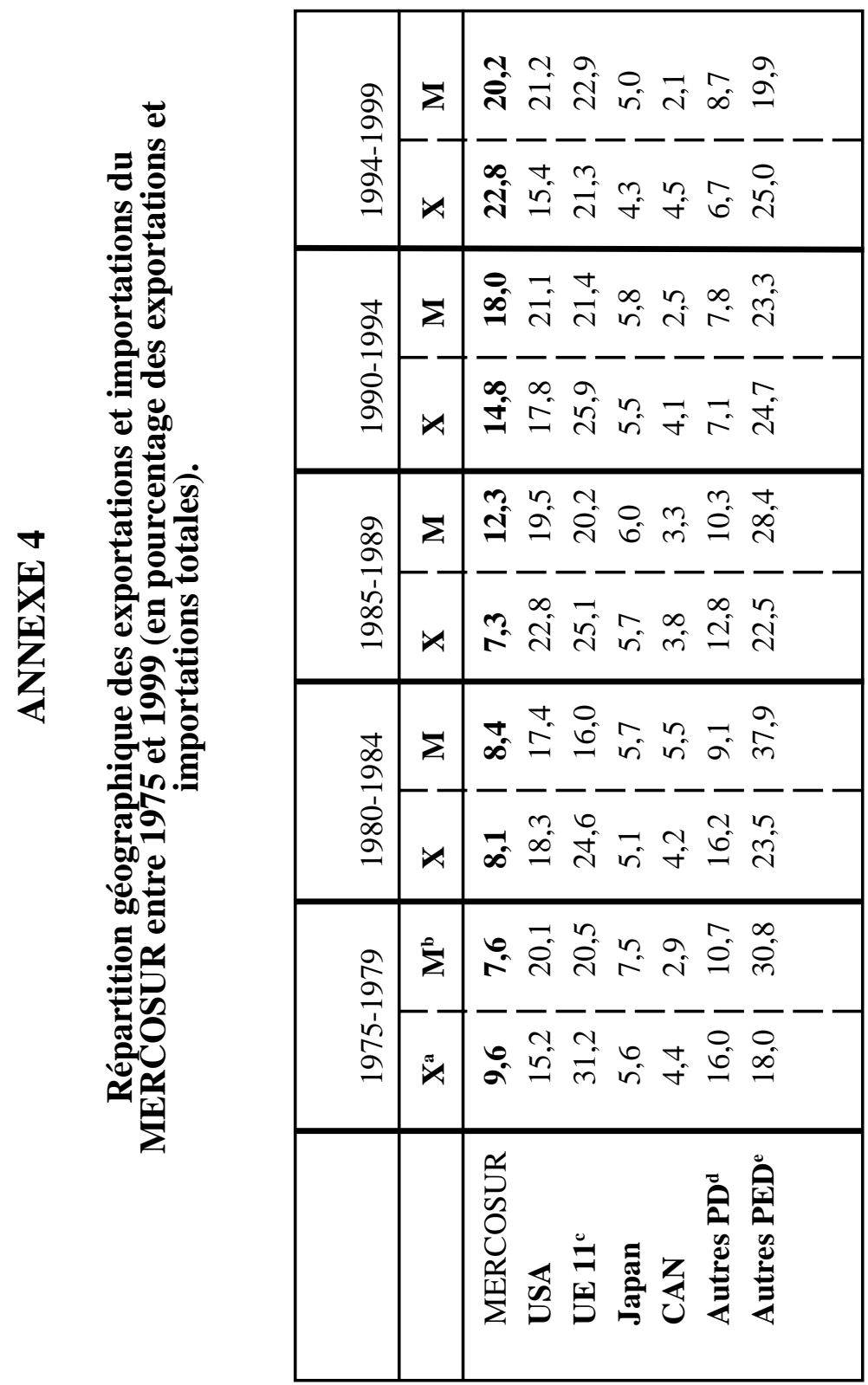




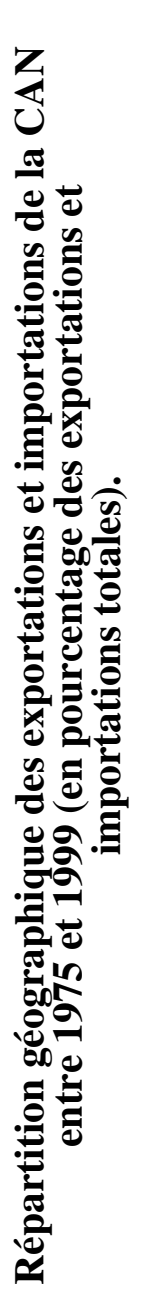

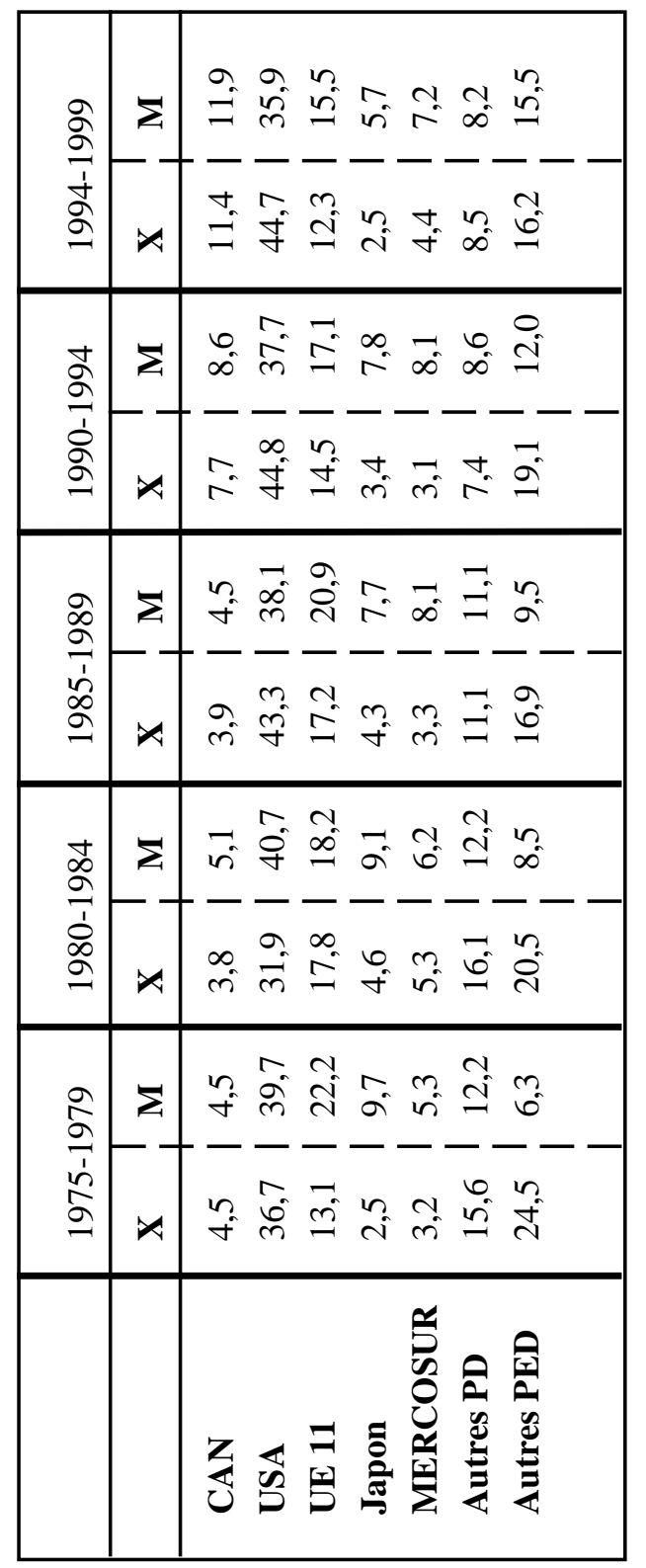

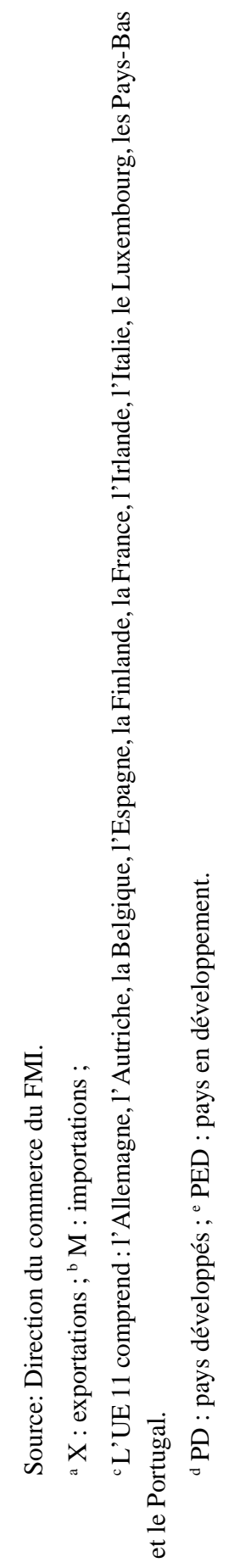




\section{ANNEXE 5}

Répartition moyenne par pays des exportations et des importations entre 19951999 (en porcentage des exportations et importations totales)

\section{EXPORTATIONS}

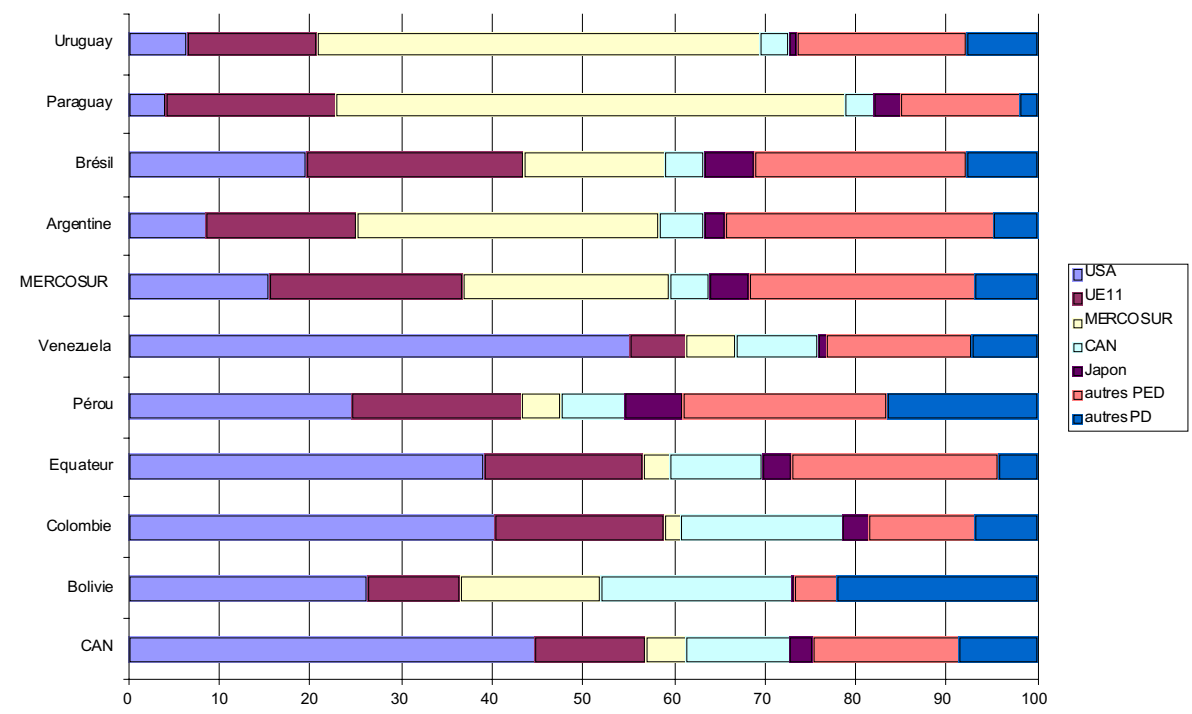

\section{IMPORTATIONS}

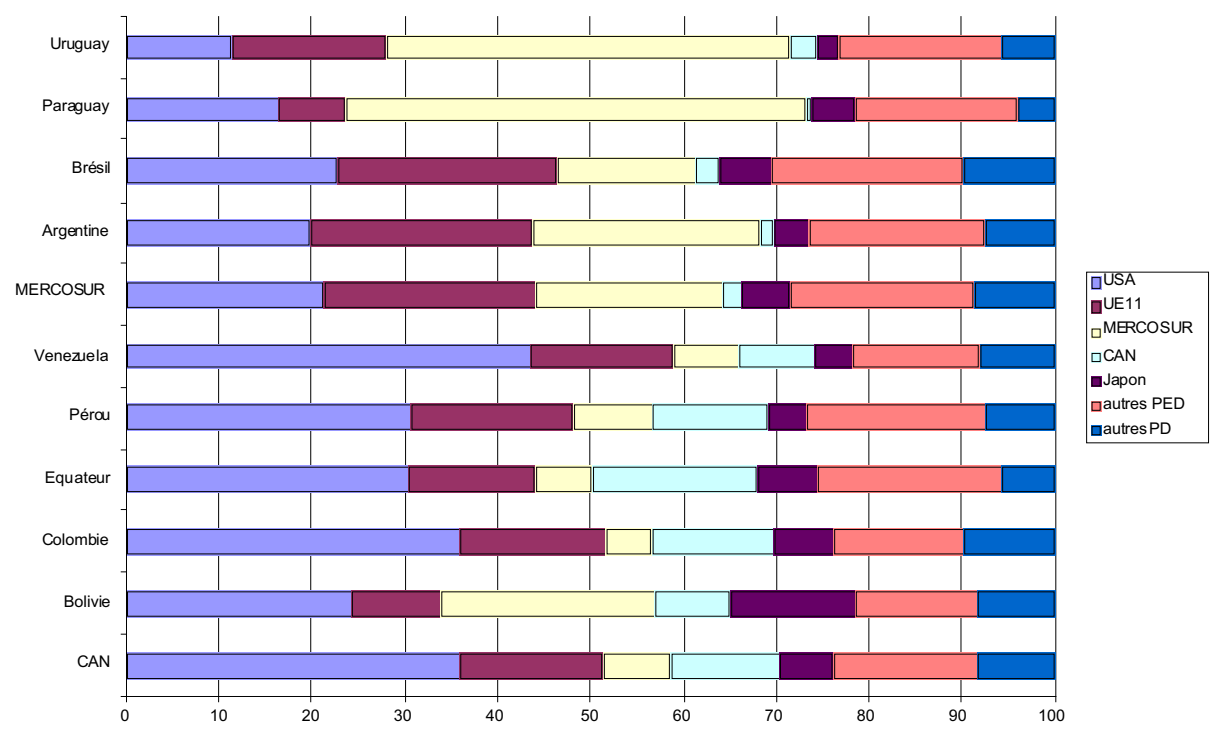

Source : Direction du commerce du FMI. 


\section{ANNEXE 6 Effets création et détournement de commerce}

Soient 3 pays A, B, et $\mathrm{C}$ et un produit $\mathrm{Z}$. A et $\mathrm{B}$ représentent les partenaires potentiels pour une ZLE ; $C$ représente le reste du monde. La demande d'importation de $\mathrm{A}\left(\mathrm{ED}_{\mathrm{A}}\right)$ indique, pour chaque prix, la quantité de bien $\mathrm{Z}$ que A désire importer. Les coûts de production $\mathrm{B}$ et $\mathrm{C}$ sont constants (1). Cette hypothèse implique que l'analyse en terme de bien être global se confonde avec celui de A. Initialement, A taxe ses importations à un taux uniforme $t$.

Alors que le cas du détournement de commerce est ambigu, celui de création ne l'est pas.

\section{EFFET CRÉATION DE COMMERCE}

Supposons que $\mathrm{B}$ ait des coûts de production inférieurs à $\mathrm{C}\left(\mathrm{P}_{\mathrm{B}}<\mathrm{P}_{\mathrm{C}}\right)$; graphiquement la courbe d'offre d'exportation de $\mathrm{C}$ (que nous ne représentons pas) est au dessus de celle de $\mathrm{B}\left(\mathrm{ES}_{\mathrm{B}}\right)$.

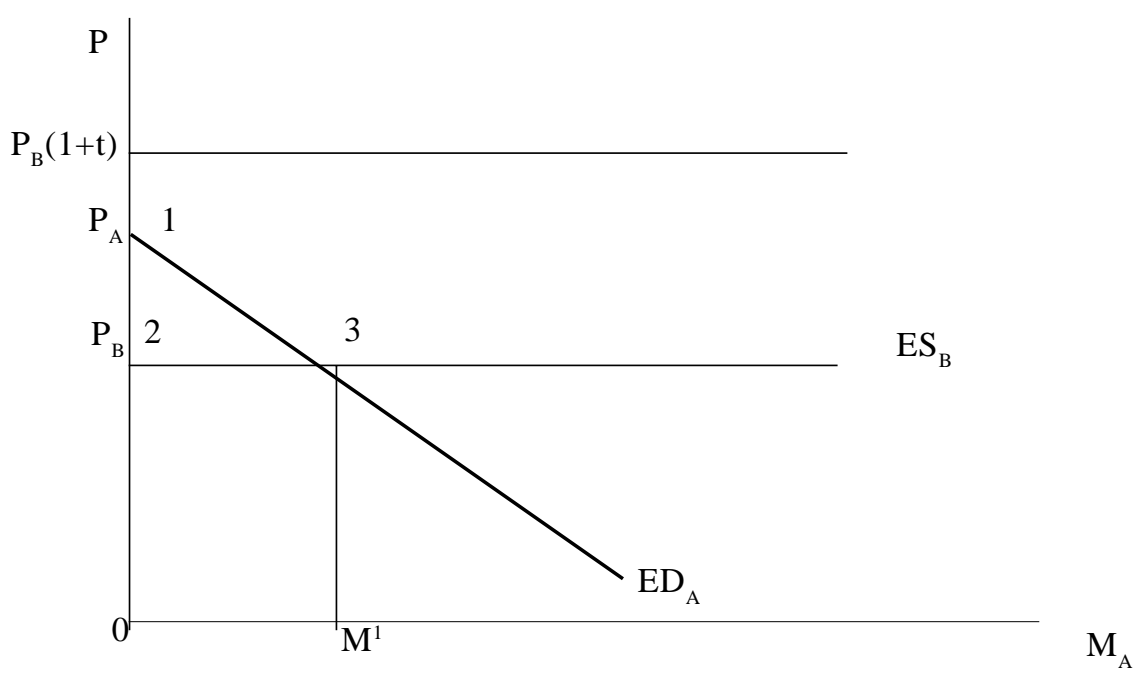

avec : $\mathrm{P}:$ prix, $\mathrm{M}_{\mathrm{A}}$ : importation de $\mathrm{A}$

ES : offre d'exportation; ED : demande d'importation ; $t$ : droit de douane

Fig. 1 : Création de commerce d'une ZLE avec coûts constants.

(1) Produire (puis exporter) une unité supplémentaire deZne modifie pas le prix ; graphiquement cela se traduit par des courbes d'offre d'exportation, $\mathrm{ES}_{\mathrm{B}}$ et $\mathrm{ES}_{\mathrm{C}}$, horizontales, c'est à dire infiniment élastique par rapport au prix. 
Avant la ZLE, comme $\mathrm{P}_{\mathrm{B}}(1+\mathrm{t})>\mathrm{P}_{\mathrm{A}}$, $\mathrm{A}$ n'importe pas $\mathrm{Z}$ et répond à toute la demande nationale en le produisant au prix $P_{A}$. Après la ZLE, les importations de $B$ ne sont plus taxées (le prix des importations pour $\mathrm{A}$ s'élève désormais à $\mathrm{P}_{\mathrm{B}}$ ). Comme $\mathrm{P}_{\mathrm{B}}<$ $\mathrm{P}_{\mathrm{A}}, \mathrm{A}$, abandonne une partie de la production de $\mathrm{Z}$ et l'importe désormais de $\mathrm{B}$ (quantité $0 \mathrm{M}^{1}$ ). La surface du triangle (123) représente l'effet création de commerce qui découle du gain d'efficacité de production de B. La ZLE entre A et B a un effet positif sur le bien être (surface 123)

\section{EFFET DÉTOURNEMENT DE COMMERCE}

Supposons que $C$ ait des coûts de production inférieurs $B\left(P_{c}<P_{B}\right)$. L'identification d'un effet de détournement de commerce part d'une situation (avant la ZLE) où $\mathrm{A}$ importe de $\mathrm{C}$ une quantité $0 \mathrm{M}^{1}$ du produit $\mathrm{Z}$ à un prix $\mathrm{P}_{\mathrm{C}}(1+\mathrm{t})$. Après la formation de la ZLE, il devient plus avantageux de s'approvisionner (pour une quantité $0 \mathrm{M}^{2}$ ) en $\mathrm{B}$ qu' en $\mathrm{C}\left(\mathrm{P}_{\mathrm{B}}<\mathrm{P}_{\mathrm{C}}(1+\mathrm{t})\right)$, bien que $\mathrm{C}$ soit plus efficient en production que $\mathrm{B}\left(\mathrm{P}_{\mathrm{C}}<\mathrm{P}_{\mathrm{B}}\right)$. Cette inefficacité productive traduit l'effet de détournement de commerce.

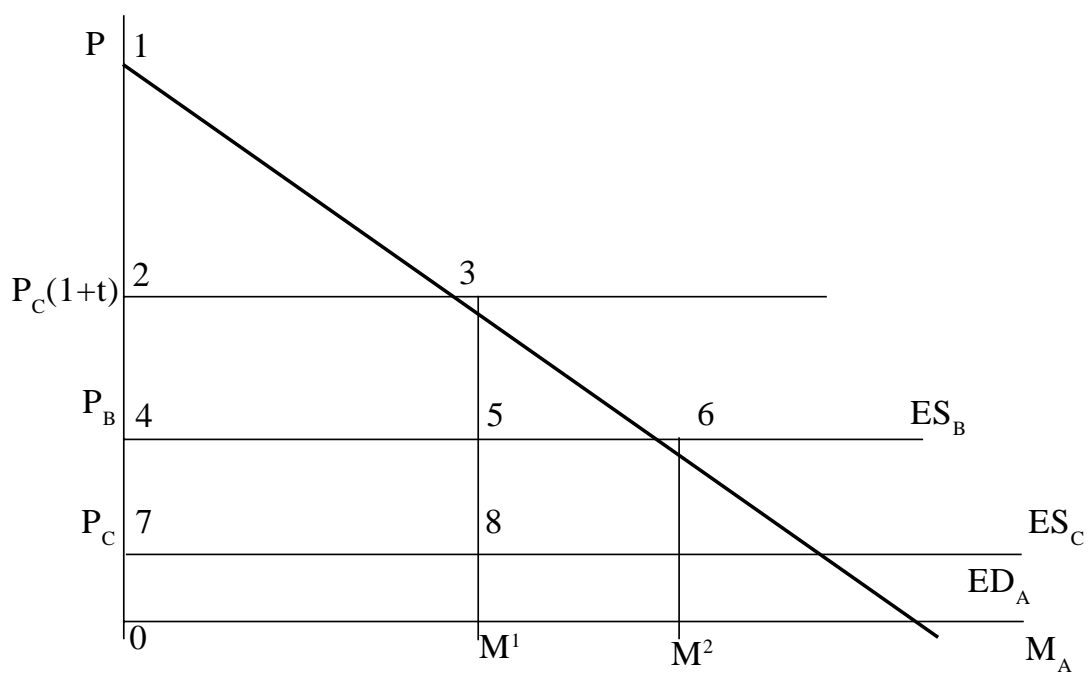

Fig. 2 : Détournement de commerce d'une ZLE avec coûts constants.

Quelle est la conséquence en terme de bien être (2) de la suppression du droit de douane entre A et B ? Avant la ZLE, le gain au commerce est représenté par la surface 1387. Après la ZLE, les recettes douanières de A (surface 2387) disparaissent. Les consommateurs de A, qui bénéficient d'un prix inférieur, voient leur bien être augmenté de la surface 2364. L'effet net sur le bien être net est la différence entre les deux surfaces

(2) L'analyse en terme de bien être repose sur l'hypothèse forte, mais traditionnellement admise par l'économie classique, selon laquelle les unités perdues et gagnées par l'État, les producteurs et les consommateurs sont équivalentes. 
(4587 - 365), le premier terme est dû à l'inefficacité de production de B et le deuxième représente le gain de consommation.

Selon la terminologie de Viner, la ZLE que nous venons de décrire effectue un détournement de commerce (approvisionnement à une source plus coûteuse en terme de coûts de production) dont l'effet négatif sur le bien être doit être comparé à l' effet positif de consommation.

À partir de ce graphique, on vérifie qu'une ZLE est susceptible d'augmenter le bien être plus : (i) l'élasticité-prix de demande d'importation est forte, (ii) le tarif douanier initial est élevé et (iii) la différence entre les coûts des pays partenaires (A et B) et des pays tiers (C) est faible.

\section{ANNEXE 7 \\ Indicateur d'avantage comparatif}

L'indicateur d'avantage comparatif répond à la question : "quels sont les points forts et les points faibles d'une économie ?"

Pour un pays (ou zone) i et chaque produit k, on calcule d'abord la part du solde observé (X-M, exportation - importation) par rapport au Produit Intérieur Brut (Y), soit en millièmes :

$$
\mathrm{y}_{\mathrm{ik}}=1000 *\left(\mathrm{X}_{\mathrm{ik}}-\mathrm{M}_{\mathrm{ik}}\right) / \mathrm{Y}_{\mathrm{i}}
$$

Le solde théorique pour le produit $\mathrm{k}$ est donné par :

$\mathrm{g}_{\mathrm{ik}} * \mathrm{y}_{\mathrm{i} .}=\left(\mathrm{X}_{\mathrm{ik}}+\mathrm{M}_{\mathrm{ik}}\right) /\left(\mathrm{X}_{\mathrm{i} .}+\mathrm{M}_{\mathrm{i} .}\right) *\left[1000 *\left(\mathrm{X}_{\mathrm{i} .}-\mathrm{M}_{\mathrm{i} .}\right) / \mathrm{Y}_{\mathrm{i}}\right]$

$\mathrm{g}_{\mathrm{ik}}$ représente la part du produit $\mathrm{k}$ dans le commerce du pays $\mathrm{i}$

$\mathrm{y}_{\mathrm{i} .}$ représente le solde commercial global par rapport à $\mathrm{Y}$

On compare alors le solde commercial observé au solde commercial théorique pour le produit $\mathrm{k}$, soit :

$\mathrm{f}_{\mathrm{ik}}=\mathrm{y}_{\mathrm{ik}}-\mathrm{g}_{\mathrm{ik}} * \mathrm{y}_{\mathrm{i}}$

sif $_{\text {ik }}>0$, le solde commercial observé est supérieur au solde commercial théorique ; identification d'un avantage comparatif ;

si fik $<0$, le solde commercial observé est inférieur au solde commercial théorique ; identification d'un désavantage comparatif . 